\title{
The X-ray-radio association in RATAN and RXTE monitoring of microquasar GRS 1915+105
}

\author{
S. A. Trushkin, ${ }^{1}$ T. Kotani, ${ }^{2}$ N. Kawai, ${ }^{3}$ M. Namiki, ${ }^{4}$ \\ and S. N. Fabrika ${ }^{5}$ \\ ${ }^{1}$ Special Astrophysical Observatory RAS, Nizhnij Arkhyz, 369167, Russia \\ ${ }^{2}$ Tokyo Tech, 2-12-1 O-okayama, Tokyo 152-8551, Japan, \\ ${ }^{3}$ Tokyo Tech, 2-12-1 O-okayama, Tokyo 152-8551, Japan \\ ${ }^{4}$ Osaka University, 1-1 Machikaneyama, Toyonaka, Osaka 560-0043, Japan \\ ${ }^{5}$ Special Astrophysical Observatory RAS, Nizhnij Arkhyz, 369167, Russia \\ email: satr@sao.ru,kotani@hp.phys.titech.ac.jp, kawai@hp.phys.titech.ac.jp, \\ namiki@ess.sci.osaka-u.ac.jp, email:fabrika@sao.ru
}

\begin{abstract}
In the daily RATAN-600 monitoring of the radio variability of the microquasar GRS1915+105 we detected a clear correlation of the flaring radio emission and X-rays "spikes" at 2-12 keV emission (1-2 Crab) detected with RXTE (ASM data) during nine bright (200-600 $\mathrm{mJy}$ ) radio flares in October 2005. The spectra of these flares in maximum were optically thick at $\nu<2.3 \mathrm{GHz}$ and optically thin at $\nu \geqslant 2.3 \mathrm{GHz}$. During radio flares the spectra of the X-ray spikes became definitely softer than those of a quiescent radio state. Thus these data indicated transitions from very high/hard states to high/soft ones during which massive ejections are probably happened, and the ejections are detected as the radio flares.
\end{abstract}

Keywords. X-rays: binaries - radio continuum: stars - radiation mechanisms: nonthermal

The X-ray transient source GRS $1910+105$ was discovered by Castro-Tirado et al. (1992) with WATCH instrument on board GRANAT. In 1994, a superluminal motion of the radio jets had been detected from GRS 1915+105 (Mirabel F. \& Rodriguez 1994). Since then a new class of astrophysical objects 'microquasars' was established. Nearly 20 microquasars were distinguished from near 300 Galactic X-ray binaries. We are far form the full understanding the jet phenomena in microquasars, however, from radio monitoring data a jet activity and state of the source can be diagnosed and even predicted.

We have carried out the 250-day almost daily monitoring observations of the microquasar GRS 1915+105 with RATAN-600 radio telescope at 1-22 GHz from September 2005 to May 2006. We have used a standard continuum radiometer complex of cryoreceivers at $4.8,7.7,11.2$, and $21.7 \mathrm{GHz}$ and the low-noise HEMT-based radiometers at 1 and $2.3 \mathrm{GHz}$. Observations were carried out using the 'Northern sector' antenna of the RATAN-600 radio telescope at the upper culmination of the sources. The measured multi-frequency light curves were directly compared with series from the X-ray observatory RXTE (ASM, Levine et al. 1996).

During the total set we have detected nine radio flares from 200 to $600 \mathrm{mJy}$, and all of them had counterparts in the soft X-rays emission (ASM RXTE). In Fig. 1 the radio and X-ray light curves in October 2005 with the first five flares are showed. The X-ray emission was defined as high (1-2.5 crabs) and hard (HR2 = 1-1.5). Radio spectra were optically thin in the first two flares, and optically thick in third one or were so-called "gigahertz-peaked" spectra, often measured from AGNs. 


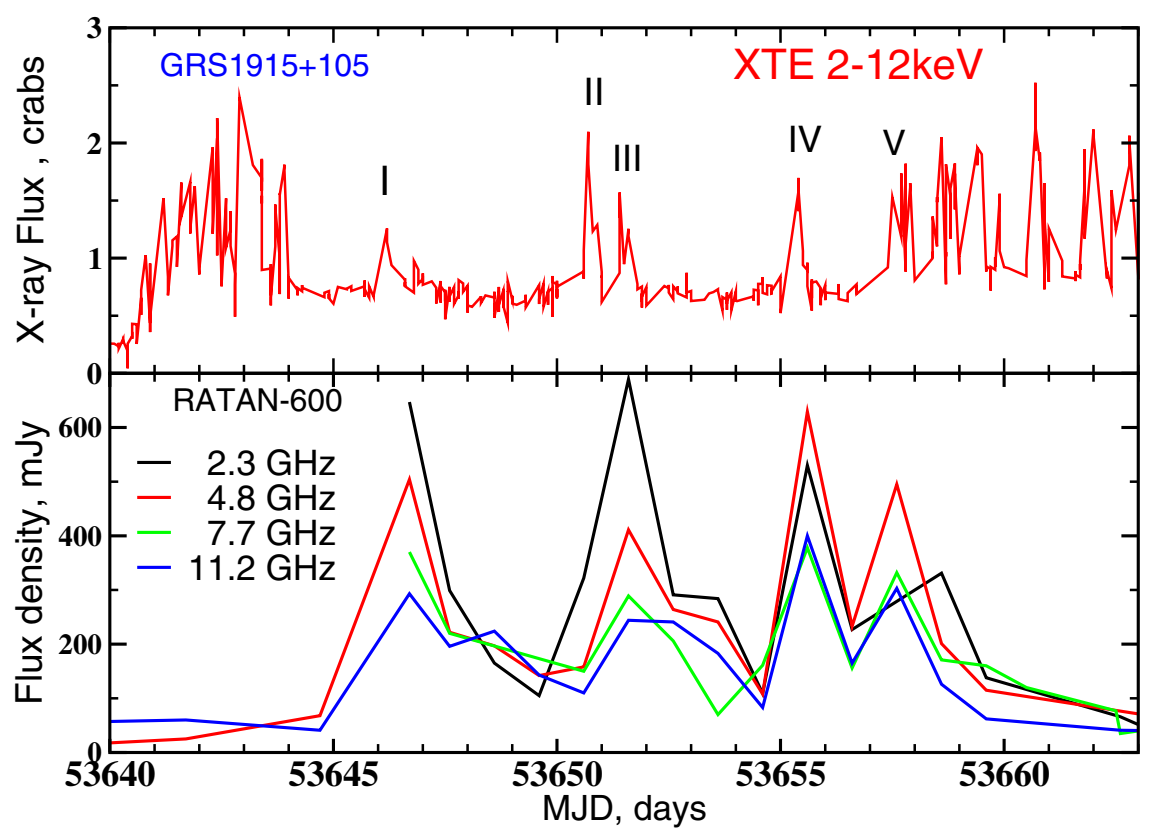

Figure 1. The radio and X-ray light curves of GRS1915+105 in October 2005.

The profiles of the X-ray spikes during the radio flares are clearly distinguishable from other spikes because of its shape, it shows the fast rise and the exponential decay. The other X-ray spikes, which reflect the activity of an accretion disk, exhibit an irregular pattern. During the radio flare, the spectra of the X-ray spikes become softer than those of the quiescent phase by a fraction of $\sim 30 \%$ in the hardness ratio $(1.3-5 \mathrm{keV} / 5-12 \mathrm{keV})$. Thus a X-ray spike associated with a radio flare has a specified characteristics, and probably only such ones could produce the massive ejections (Namiki et al. 2007).

Miller-Jones et al. (2006) have detected a large-scale radio jet with VLBA mapping during the ninth radio outburst on 23 February 2006 (MJD53789.258), associated with a X-ray spike. Then the optically thin flare with fluxes $340,340,342,285,206$, and 153 mJy was detected at frequencies 1, 2.3, 4.8, 7.7, 11,2 and 21.7 GHz.

\section{Acknowledgments}

These studies were supported by Russian Foundation Base Research (RFBR) grant N 05-02-17556 and mutual RFBR and Japan Society for the Promotion of Science (JSPS) grant N 05-02-19710.

\section{References}

Levine, A., Bradt, H. \& Cui, W. 1996, ApJ, 469, L33

Castro-Tirado, A. J., Brandt, S. \& Lund, N. 1992, IAUC, 5590

Mirabel, I. F. \& Rodriguez, L. F. 1994, Nature, 371, 46

Miller-Jones, J. C. A., Rupen, M. P. \& Trushkin, S. 2006, ATel, 758, 1

Namiki, M., Trushkin, S. \& Bursov, N. 2007, in VI microquasars workshop, Komo, Italy, PoS, (in press) 